\title{
$\mathrm{C}_{60}^{-}$离子 $\pi$ 电子云特性的核磁共振研究
}

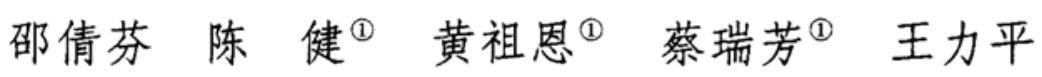 \\ (复旦大学分析测试中心; (1) 复旦大学化学系, 上海 200433)
}

\section{关键词 $\mathrm{K}_{1} \mathrm{C}_{60}(\mathrm{THF})$ 核磁共振 电子云特性}

由于 $\mathrm{C}_{60}$ 具有奇特的球形笼状结构以及不寻常的物理、化学性质, 所以 $\mathrm{C}_{60}$ 及其衍生物成 了当前物理、化学及材料科学等各个学科研究的前沿课题, 特别是集中于 $\mathrm{A}_{x} \mathrm{C}_{60}$ 这类化合物的 研究, 因为在 $\mathrm{K}_{3} \mathrm{C}_{60}, \mathrm{Rb}_{3} \mathrm{C}_{60}$ 及 $\mathrm{Rb}_{2} \mathrm{CsC}_{60}$ 等化合物里测到了使人感兴趣的超导电性. 但是在对 $\mathrm{A}_{x} \mathrm{C}_{60}$ 等 $\mathrm{C}_{60}$ 负离子盐的核磁共振 (NMR) 研究中, 有一个反常的现象始终困扰着研究者. 纯 $\mathrm{C}_{60}$ 无论是晶形粉末还是在苯、甲苯中的溶液, 用 NMR 测得的化学位移 $\delta$ 均为 143 (相对于 TMS), -而碱金属掺杂的 $\mathrm{K}_{3} \mathrm{C}_{60}$ 的 ${ }^{13} \mathrm{C}$ 谱线中心位移 $8186^{[1]}$, 溶液中抗磁性 $\mathrm{C}_{60}^{-n}(n$ 可能为 6) 的化学位移为 $157^{[2]}$. 显然, 这种实验现象与对传统的化学位移的认识产生了矛盾. 化学位移理论认为, 核周 围空间电子云的分布使外磁场中的原子核受到电子云屏蔽, 对抗磁性的核来说, 化学位移应 向高场移动, 而 $\mathrm{C}_{60}^{n-}$ 的化学位移 (相对于中性的 $\mathrm{C}_{60}$ ) 却向低场移动.

1993 年, 美国贝尔实验室的 Pasquarello 等人首先提出了新的环电流模型来解释这一现象 ${ }^{[3]}$. 他们认为: 在 $\mathrm{C}_{60}$ 分子中, 环电流可按球内、外表面分成两部分, 它们分别由内层和外层 $\pi$ 电子 云感应所致. 这两部分电子云产生的环电流感应对化学位移的贡献各异, 例如 $\mathrm{C}_{60}^{6-}$ 中, 当电子 云对称地分布在球内、外两侧时, 环电流感应的化学位移计算值为负值, 即化学位移 $\delta$ 向高场 移动; 但若 $\pi$ 电子云不对称地向球外侧作较小的位移, 则足以产生较大的由环电流感应的正 向化学位移, 亦即 $\delta$ 向低场移动. 因此, Pasquarello 等人认为 $\pi$ 电子云不对称位移是理解 $\mathrm{C}_{60}^{n-}$ 化学位移实验现象的关键. 我们关于 $\mathrm{K}_{1} \mathrm{C}_{60}(\mathrm{THF})_{x}(0<x<1)$ 化合物的核磁共振研究进一 步支持了上述观点.

\section{1 实验}

为了深人研究 $C_{60}$ 衍生物电子云的特点与化学位移之间的关系, 我们制备了在 $C_{60}^{-1}$ 表面 $\pi$ 电子云分布不同的两种 $\mathrm{K}_{1} \mathrm{C}_{60}(\mathrm{THF})$ 分子. 由于样品对空气、水汽极其敏感, 因此所有的制样 操作均在无水无氧条件下进行. $\mathrm{K}_{1} \mathrm{C}_{60}$ (THF) 从四氢呋喃溶液中制备 (具体过程及波谱学表 征另文发表), 系黑色晶形的富勒烯盐, 称为样品 A. 然后取其一半在已烷中摚拌约 $1 \sim 2 \mathrm{~h}$ 后, 室温下进一步抽真空约 $1 \mathrm{~h}$, 得 $\mathrm{K}_{1} \mathrm{C}_{60}(\mathrm{THF})_{x}(0<x<1)$, 呈黑色粉末状, 称为样品 $\mathrm{B}$. 将样 品 $\mathrm{A}, \mathrm{B}$ (各 $200 \mathrm{mg}$ 左右) 封人高纯氯气保护的样品管中, 作 NMR 测试. 使用的仪器为 德国BRUKER 公司的 MSL-300 超导核磁共振仪 (相应的 ${ }^{13} \mathrm{C}$ 的共振频率为 $75.468 \mathrm{MHz}$ ), ${ }^{13} \mathrm{C}$ 的

\footnotetext{
1994-06-22 收稿, 1994-12-21 收修改稿
} 
$90^{\circ}$ 脉宽为 $8.5 \mu \mathrm{s}$. 谱信号由 FID 信号经 FT 变换而得, 线宽利用 Linesim 程序模拟读得. 自 旋 - 晶格驰豫时间 $T_{1}$ 系用非周期脉冲序列 (APS) 的饱和恢复法. 弛豫曲线用单指数拟合, 吻 合较好.

\section{2 结果和讨论}

样品 A, B 室温下的 ${ }^{13} \mathrm{C}$ NMR 谱如图 1 所示. 样品 $\mathrm{A}$ 只有一个单峰, 化学位移 $\delta_{\mathrm{A}}=187$ (为比较起见, 图中还示出了纯 $\mathrm{C}_{60}$ 粉末 ${ }^{13} \mathrm{C}$ 谱, $\delta=143$ (相对于 TMS)), 线宽 $\Delta v_{\mathrm{A}}=1.22 \mathrm{kHz}$. 样 品 $\mathrm{B}$ 有两个峰, 其中峰 $\mathrm{B}_{1}$ 与峰 $\mathrm{A}$ 很类似, $\delta_{\mathrm{B}_{1}}=187$, 线宽 $\Delta v_{\mathrm{B}_{1}}=1.29 \mathrm{kHz}$; 而另一个峰 $\mathrm{B}_{2}$ 与峰 $\mathrm{A}$ 相差甚大, $\delta_{\mathrm{B}_{2}}=162, \Delta v_{\mathrm{B}_{2}}=0.76 \mathrm{kHz}$. 此外, 样品 $\mathrm{A}$ 的自旋 -晶格弛豫时间 $T_{1(\mathrm{~A})}=15.9 \mathrm{~ms}$, 样品 $\mathrm{B}$ 的第一个峰 $T_{1\left(\mathrm{~B}_{1}\right)}=14.4 \mathrm{~ms}$, 第二个峰 $T_{1\left(\mathrm{~B}_{2}\right)}=134.0 \mathrm{~ms}$.

根据变温顺磁共振 (ESR) 的研究, 我们认为样品 $\mathrm{B}$ 的 2 个峰分别代表了 $\mathrm{C}_{60}^{-}$离子因 Jahn-Teller 效应而产.生的两种电子态 (详细内容另文发表). 在样品 $\mathrm{A}$ 中, $\mathrm{C}_{60}^{-}$与 $\mathrm{K}^{+}$形成离子 型分子, 四氢呋喃 $(\mathrm{THF})$ 分子通过配位键与 $\mathrm{K}^{+}$结合 $\left(\mathrm{THF}\right.$ 与 $\mathrm{K}^{+}$的配位在一定程度上阻止了 $\mathrm{K}^{+}$和其他邻近 $\mathrm{C}_{60}^{-}$的静电吸引力) 在同一个 $\mathrm{K}_{1} \mathrm{C}_{60}(\mathrm{THF})$ 分子中, $\mathrm{C}_{60}^{-}$与 $\mathrm{K}^{+}$之间存在较强的静 电吸引力, $\mathrm{C}_{60}^{-}$与 $\mathrm{K}^{+}$为紧密离子对. 而样品 $\mathrm{B}$, 由于 $\mathrm{THF}$ 被抽去一部分, 因而部分离子型分 子 $\mathrm{K}_{1}^{+} \mathrm{C}_{60}^{-}$不再结合 $\mathrm{THF}$; 这些分子里的 $\mathrm{K}^{+}$与邻近分子中的 $\mathrm{C}_{60}^{-}$之间的静电吸引力增强, 而和

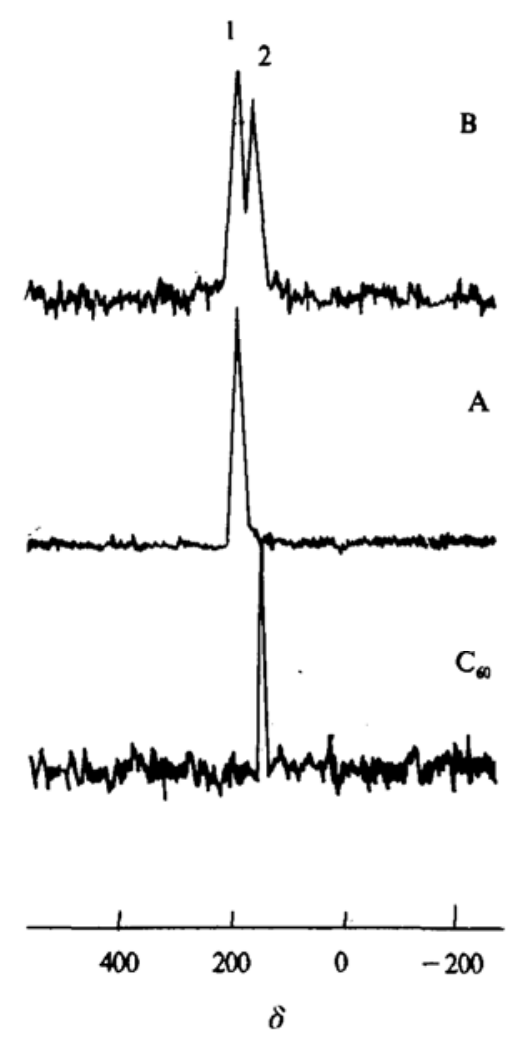

图 1 样品 $\mathrm{A}, \mathrm{B}$ 室温下的 ${ }^{13} \mathrm{C} \mathrm{NMR}$ 谱图

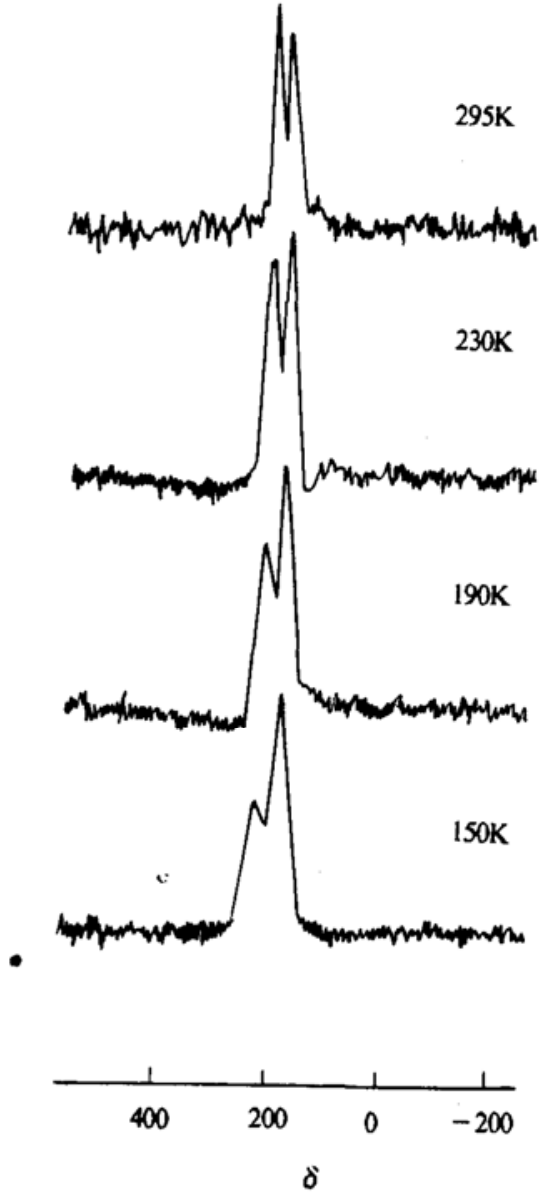

图 2 样品 $\mathrm{B}$ 不同温度 ${ }^{13} \mathrm{C} \mathrm{NMR}$ 谱图 
同一分子内自己的 “伙伴” $\mathrm{C}_{60}^{-}$的结合力减弱, 所以这类分子呈松散的离子对 $\mathrm{C}_{60}^{-} \cdots \mathrm{K}^{+}$, 而样品 $\mathrm{B}$ 里另一部分未抽去 THF 的分子, 性质仍类似于样品 A. 按 Pasquarello 环电流模型, $\mathrm{C}_{60}^{-}$的化学 位移 $\delta_{\mathrm{A}}, \delta_{\mathrm{B}_{1}}$ 和 $\delta_{\mathrm{B}_{2}}$ 相对于 $\mathrm{C}_{60}$ 朝低场位移表明, $\mathrm{C}_{60}^{-}$中 $\pi$ 电子云不对称地分布于球的内、外表面 (即电子云向外侧位移). $\delta_{\mathrm{A}},\left(\delta_{\mathrm{B}_{1}}\right)$ 与 $\delta_{\mathrm{B}_{2}}$ 的差异反映了紧密离子对 $\mathrm{C}_{60}^{-}-\mathrm{K}^{+}$和疏松离子对 $\mathrm{C}_{60}^{-} \cdots \mathrm{K}^{+}$中 $\mathrm{C}_{60}^{-} \pi$ 电子云不对称程度的不同, 即前者 $\mathrm{C}_{60}^{-} \pi$ 电子云向外侧位移的程度较后者大, 这差异又归因于两者 $\mathrm{K}^{+}$与 $\mathrm{C}_{60}^{-}$离子间库仑力的不同. 上述差异同样反映在核自旋 - 晶格弛 豫时间 $T_{1}$ 上. 按 Tycko 等人的观点, $\mathrm{A}_{x} \mathrm{C}_{60}$ 的弛豫机制不是由化学位移各向异性引起, 而是由 偶极超精细耦合所致 ${ }^{[4]}$.

图 1 中 $B_{1}$ 峰与 $B_{2}$ 峰的面积之比近似为 $1.3: 1$, 所以 $x$ 值约为 0.57 . 为了验证上述的内容, 我们曾经把样品 $\mathrm{B}$ 的抽空与搅拌时间加长, 使 THF 含量进一步减少, 即 $x$ 值变小经 NMR 测 试, 获得另一相似的谱图 (为节省篇幅, 未附), 这时 $\mathrm{B}_{2}$ 峰的相对面积增大, 与理论推测一致.

我们还对样品 B 进行了变温测试, 温度从 $295 \mathrm{~K}$ 下降到 $150 \mathrm{~K}$, 其线宽与化学位移随温度 的变化值列于表 1. 其中 $295,230,190,150 \mathrm{~K}$ 温度下的 ${ }^{13} \mathrm{C}$ NMR 谱如图 2 所示. 由表 1 可知: 1) 随着温度降低, 紧密离子对中的 $\mathrm{C}_{60}^{-}$离子旋转变慢的程度 (峰 1) 较疏松离子对的 (峰 2) 要大, 这清楚地反映在线宽随温度变化的结果. 2) 随着温度的降低, 紧密离子对中 $\mathrm{K}^{+}$和 $\mathrm{C}_{60}^{-}$的静电 吸引力增强, 因而 $\pi$ 电子云向球分子外侧的动态位移加剧, 导致感应正向化学位移的环电流 增强, 所以化学位移值明显地移向低场 (峰 1 ), 而疏松离子对中 $\mathrm{C}_{60}^{-}$离子的 $\pi$ 电子云受温度变 化的影响不大 (峰 2). 由谱模拟显示, 不同温度下两个峰所包括的相对面积比基本相同.

表 1 变温条件下样品 $\mathrm{B}$ 两个峰的 $\delta$ 和 $\Delta v$

\begin{tabular}{lcccccccccc}
\hline 温度 $/ \mathrm{K}$ & 295 & 270 & 260 & 250 & 240 & 230 & 210 & 190 & 170 & 150 \\
\hline 峰 1 化学位移 $\delta$ & 187 & 189 & 194 & 195 & 195 & 195 & 197 & & 201 & 209 \\
峰 2 化学位移 $\delta$ & 162 & 159 & 162 & 162 & 162 & 162 & 161 & & 163 & 159 \\
峰 1 线宽 $\Delta v / \mathrm{kHz}$ & 1.2 & 1.4 & 1.5 & 1.7 & 1.7 & 2.1 & 2.0 & 2.5 & 3.0 & 3.9 \\
峰 2 线宽 $\Delta v / \mathrm{kHz}$ & 0.7 & 0.7 & 0.8 & 0.9 & 1.0 & 1.3 & 1.5 & 2.0 & 2.3 & 2.6 \\
\hline
\end{tabular}

总之, 我们利用 Pasquarello 的环电流模型和固体核磁共振技术, 发现 $\mathrm{C}_{60}^{-}$离子 $\pi$ 电子云特 性与其化学位移之间存在着很强的关联性.

\section{参考文献}

1 Tycko R, Dabbagh G, Rosseinksy $M$ J et al. ${ }^{13} \mathrm{C}$ NMR spectroscopy of $\mathrm{K}_{\mathrm{x}} \mathrm{C}_{\theta 0}$ : phase secaration, molecular dynamics and metallic properties. Science, 1991, 253: 884 886

2 Bausch J W, Surya G K, Olah G A. Diamagnetic polyanions of the $\mathrm{C}_{\infty 0}$ and $\mathrm{C}_{0}$ fullerenes: preparation, ${ }^{13} \mathrm{C}$ and ${ }^{17} \mathrm{Li}$ NMR spectroscopic observatin and alkylation with methyl iodide to polymethylated fullerenes. J Am Chem Soc, 1991, 113: $3205 \sim 3206$

3 Pasquarello A, Schluter M, Haddon R C. Ring currents in topologically complex molecules: Application to $\mathrm{C}_{60}, \mathrm{C}_{x 0}$, and their hexa-anions. Physical Review A, 1993, 47:1783 1 789

4 Tycko R, Dabbagh G, Murphy D W et al. Electronic properties and phase transitions of $\mathrm{RbC}_{60}$ and $\mathrm{CsC}_{60}$ : investigation by NMR spectrosoopy. Physical Review B, 1993, 48: $9097 \sim 9105$ 